\title{
SARS-CoV-2 Variants and Vaccination
}

\author{
Yang Liu' ${ }^{1, *}$, Jianying Liu' ${ }^{1}$ and Pei-Yong Shi,3,4,5,6,*
}

\begin{abstract}
Severe acute respiratory syndrome coronavirus 2 (SARS-CoV-2) has caused global destruction since its emergence in late 2019. Over the past 2 years, the virus has continually evolved in human hosts, thus leading to the emergence of variants with altered viral transmission, disease severity, and evasion of immunity. Although vaccines for coronavirus disease 2019 (COVID-19) have been developed at an unprecedented pace, the emergence of variants has posed a constant threat to the effectiveness of the approved vaccines. In this Commentary, we review the key variants and discuss their implications in viral replication, transmission, and immune evasion.
\end{abstract}

Key words: SARS-CoV-2 variants, spike mutations, neutralization, vaccine, COVID-19
Severe acute respiratory syndrome coronavirus 2 (SARS-CoV-2), the pathogen responsible for coronavirus disease 2019 (COVID-19), has caused a global pandemic since its emergence in Wuhan, China in late 2019 [1]. As of January 5, 2022, it has caused more than 296 million infections leading to more than 5.4 million fatalities [2]. As a positive single-stranded RNA virus whose polymerase lacks proofreading capability, SARS-CoV-2 frequently mutates during viral replication, thus generating numerous viral variants with altered viral infectivity, transmission, and disease severity [3]. The original Wuhan isolate sequence of the spike glycoprotein on SARS-CoV-2, which binds the cellular receptor angiotensin converting enzyme 2 (ACE2), was used for vaccine development. New mutations in the spike gene have continually emerged in variants over time. This Commentary (i) summarizes the key variants and their spike mutations, and (ii) discusses their implications in viral replication, transmission, and immune evasion.

\section{DOMINANT SARS-COV-2 VARIANTS}

Among all SARS-CoV-2 strains, some notable variants have fitness advantages and have outcompeted other variants during viral evolution. The first dominant spike (S) protein D614G substitution increases viral replication in the human upper respiratory tract; this mutant rapidly replaced nearly all prior SARS-CoV-2 strains from June 2020 onward [4]. Subsequently, the Alpha variant (B.1.1.7 lineage) first reported in the United Kingdom and quickly spread to many parts of the world, owing to its increased binding affinity for human ACE2 receptor [5-7]. Next, the Delta variant (B.1.617.2 lineage) first reported in India in October 2020, spread rapidly, and displaced the Alpha variants worldwide [8-10]. Recently, the heavily mutated Omicron variant, first detected in South Africa in November 2021, has explosively spread to many countries and led to global Omicron surges [11-13]. According to previous studies on known mutations and clinical
Edited by:

Hua Li, Hubei Key Laboratory of Natural Medicinal Chemistry and Resource Evaluation, School of Pharmacy, TongjiRongcheng Center for Biomedicine, Tongji Medical College, Huazhong University of Science and Technology

Reviewed by:

Chengfeng Qin, State Key Laboratory of Pathogen and Biosecurity, Institute of Microbiology and Epidemiology, Academy of Military Medical Sciences, China

Xionglin Fan, School of Basic Medical Sciences, Huazhong University of Science \& Technology

${ }^{*}$ Corresponding authors:

E-mail: liuyang.fdu@hotmail.com (YL); peshi@UTMB.edu (P-YS)

${ }^{1}$ Institute of Infectious Disease, Shenzhen Bay Laboratory, Shenzhen, Guangdong, China

${ }^{2}$ Department of Biochemistry and Molecular Biology, University of Texas Medical Branch, Galveston, TX, USA ${ }^{3}$ Institute for Human Infections and Immunity, University of Texas Medical Branch, Galveston, TX, USA

${ }^{4}$ Institute for Translational Sciences, University of Texas Medical Branch, Galveston, TX, USA

${ }^{5}$ Sealy Institute for Drug Discovery, University of Texas Medical Branch, Galveston, TX, USA

${ }^{6}$ Sealy Institute for Vaccine Sciences, University of Texas Medical Branch, Galveston, TX, USA

Received: January 62022

Revised: January 252022

Accepted: January 262022

Published Online: February 82022 
observations, the Omicron variant is transmitted much more rapidly than the Delta variant, with a case doubling time as short as 1.5-3 days. In addition, Omicron can escape many monoclonal antibodies and evade vaccine-elicited neutralization [14-16]. Furthermore, infection with previous non-Omicron variants does not appear to elicit robust neutralization against Omicron [17]. However, booster vaccination (e.g., a third dose of the Pfizer/BioNTech vaccine or a booster dose of the Moderna vaccine) elicits good neutralization against Omicron [18], thus supporting a booster vaccination strategy. The durability of the protective immunity of booster vaccination against Omicron remains to be determined. Omicron's high transmissibility and immune evasion have enabled it to become the new dominant SARS-CoV-2 variant. More than 1 million cases in a single day have been recorded in the United States (Fig 1).

\section{NOTABLE MUTATIONS IN THE S PROTEIN}

The trimeric S glycoprotein of coronaviruses is the major surface protein present on the viral envelope [19]. Mature S protein, formed through cleavage by furin and transmembrane serine protease 2 (TMPRSS2), mediates viral binding to the ACE2 receptor, entry, and immune escape. Thus, S protein is considered the key determinant of viral infectivity and transmissibility [20]. The D614G substitution is the first dominant mutation that occurred in the $\mathrm{S}$ protein. Elevated quantities of D614G virus have been detected in the respiratory tracts in both patients and animal models, thus indicating this mutant's higher infectivity and transmissibility [21,22]. Another mutation, N501Y, located at the receptor-binding domain in the $\mathrm{S}$ protein, has been demonstrated to be the most important amino acid substitution in the Alpha variant. The N501Y substitution markedly increases the binding affinity to the human ACE2 receptor, thus leading to rapid infection in the upper respiratory tract and higher viral transmission $[7,23]$. The Delta variant has the spike mutation
P681R, which is located at a furin-cleavage site in the S protein. Experimental results in human primary airway epithelial culture and animal models have demonstrated that the P681R substitution enhances viral replication and infection, possibly through increasing the furin cleavage of the full-length S protein into S1 and S2 subunits [10,24]. Many other amino acid changes in the $\mathrm{S}$ protein, including S13I, L18F, 69-70 deletion, W152C, K417N/T, N439K, N440K, L452R, Y453F, S477G/N/R, E484K/Q/P, S494P, and $\mathrm{H} 655 \mathrm{Y}$, have been reported to decrease vaccinated serum neutralization and monoclonal-antibody inhibition, and/ or increase viral infection and transmission (Table 1). The Omicron variant contains more than 30 mutations in the $\mathrm{S}$ protein, several of which are present in other variants of concern, including Alpha, Beta, Gamma, and Delta [39]. Some of these mutations, including 69-70 deletion, K417N, N440K, S477N, E484A, N501Y, D614G, H655Y, and P681H, have been well studied and are known to enhance viral infectivity, transmissibility, and immune escape, thus leading to high concern regarding the pandemic's potential severity resulting from ongoing Omicron surges.

\section{NEUTRALIZATION OF SARS-COV-2 VARIANTS AND VACCINE STRATEGIES}

Several COVID-19 vaccines have been approved and used for immunization globally to develop herd immunity against COVID-19 [40]. The Pfizer/BioNTech BNT162b2 nucleoside-modified mRNA vaccine is one of the most common vaccines, which has been widely used in North America and Europe [41]. Because of the high mutation frequency of SARS-CoV-2 S protein, some newly emerged variants have diminished susceptibility to neutralization by antibodies generated by vaccination or natural infection. Several approaches have been used to measure the neutralization sensitivity of variants to human sera, including pseudotype virus for expression of SARS-CoV-2 S protein, clinical viral

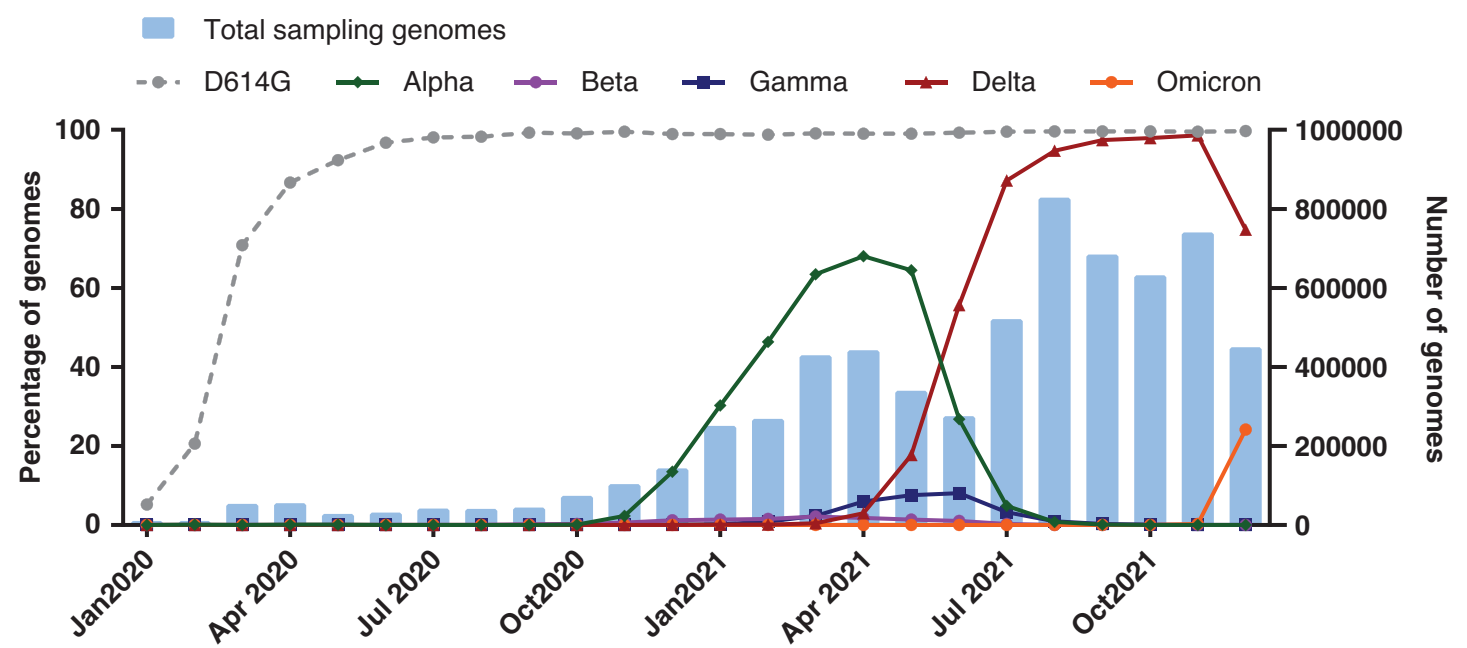

FIGURE 1 | Frequencies of the D614G, Alpha, Beta, Gamma, Delta, and Omicron lineages over time in all genomic SARS-CoV-2 sequences. Blue bars represent the total numbers of SARS-CoV-2 genomes. Colored lines indicate the percentages of different lineages in the total SATSCoV-2 genome. All data were sourced from the GISAID database from January 2020 to December 2021. 
TABLE 1 | Notable mutations increasing SARS-CoV-2 infectivity, transmissibility, and immune escape.

\begin{tabular}{|c|c|c|c|c|}
\hline Mutations & Locations & Representative Lineages & Functions & References \\
\hline S13। & $\mathrm{N}$-terminal domain & B. 1.427 & Immune escape & {$[25]$} \\
\hline L18F & $\mathrm{N}$-terminal domain & Beta, Gamma & Immune escape & {$[26]$} \\
\hline del69-70 & $\mathrm{N}$-terminal domain & Alpha, Omicron * & Immune escape/increased transmissibility & {$[27]$} \\
\hline W152C & $\mathrm{N}$-terminal domain & B.1.427 & Immune escape & {$[25]$} \\
\hline $\mathrm{K} 417 \mathrm{~N} / \mathrm{T}$ & Receptor-binding domain & Beta, Gamma, Omicron & Attenuated biding affinity to ACE2/immune escape & {$[28]$} \\
\hline N439K & Receptor-binding domain & AV.1 & Increased binding affinity to ACE2/immune escape & [29] \\
\hline N440K & Receptor-binding domain & Omicron & Increased infectivity & {$[30]$} \\
\hline L452R & Receptor-binding domain & Delta & Immune escape & [31-33] \\
\hline Y453F & Receptor-binding domain & Delta & Immune escape & {$[34]$} \\
\hline S477G/N/R & Receptor-binding domain & $\underline{\text { Omicron }}$ & Immune escape & {$[35]$} \\
\hline $\mathrm{E} 484 \mathrm{~K} / \mathrm{Q} / \mathrm{P}$ & Receptor-binding domain & $\begin{array}{l}\text { Beta, Gamma, Delta, Eta, Lota, } \\
\text { Kappa, Mu, Omicron }\end{array}$ & Immune escape & {$[31,32]$} \\
\hline S494P & Receptor-binding domain & Alpha & Immune escape & {$[36,37]$} \\
\hline N501Y & Receptor-binding domain & Alpha, Beta, Gamma, Mu, Omicron & Increased infection and transmissibility & {$[7,28]$} \\
\hline D614G & The C-terminal of S1 domain & All lineages & Increased infectivity & {$[22]$} \\
\hline $\mathrm{H} 655 \mathrm{Y}$ & Near S1/S2 cleavage site & Gamma, Omicron & Immune escape & [38] \\
\hline $\mathrm{P} 681 \mathrm{H} / \mathrm{R}$ & Near S1/S2 cleavage site & Alpha, Kappa, Mu, Omicron & Increased cleavage efficiency & {$[10]$} \\
\hline
\end{tabular}

*Omicron is underlined to indicate multiple mutations and deletions with respect to other variants.

isolates, and chimeric SARS-CoV-2 bearing the $\mathrm{S}$ protein from different variants $[13,14,42]$. Among these approaches, the use of chimeric SARS-CoV-2 bearing variant $\mathrm{S}$ has two major advantages. First, this approach does not require waiting for the isolation of clinical viral strains; as soon as the $\mathrm{S}$ sequence is available, the variant $\mathrm{S}$ sequence can be synthesized and engineered into the original SARS-CoV-2 backbone [43]. Second, in this approach, in contrast to the pseudovirus approach, the chimeric virus is an authentic SARS-CoV-2. The 50\% plaque-reduction neutralization titer against various recombinant viruses can be easily tested and accurately compared among all variants. The Alpha variants have higher infection and transmission efficiency, but the neutralization titers are approximately equivalent to those of the WT strain [42]. The D614G and representative Delta variants exhibit modestly lower neutralization titers than the WT virus $[44,45]$. Unfortunately, two doses of the Pfizer vaccine are insufficient to induce robust antibody neutralization against the Omicron variant [46]. The above studies were all performed on the same set of human sera collected 2 or 4 weeks after the administration of two doses of the Pfizer vaccine (Fig 2). Many other studies have also demonstrated that the neutralization titers of infected and vaccinated people are significantly lower against Omicron than the WT virus $[17,18]$. However, as described above, a third dose of the Pfizer vaccine increases the magnitude and breadth of neutralization, thus leading to robust neutralization against the Omicron variant $[18,46,47]$. To date, the

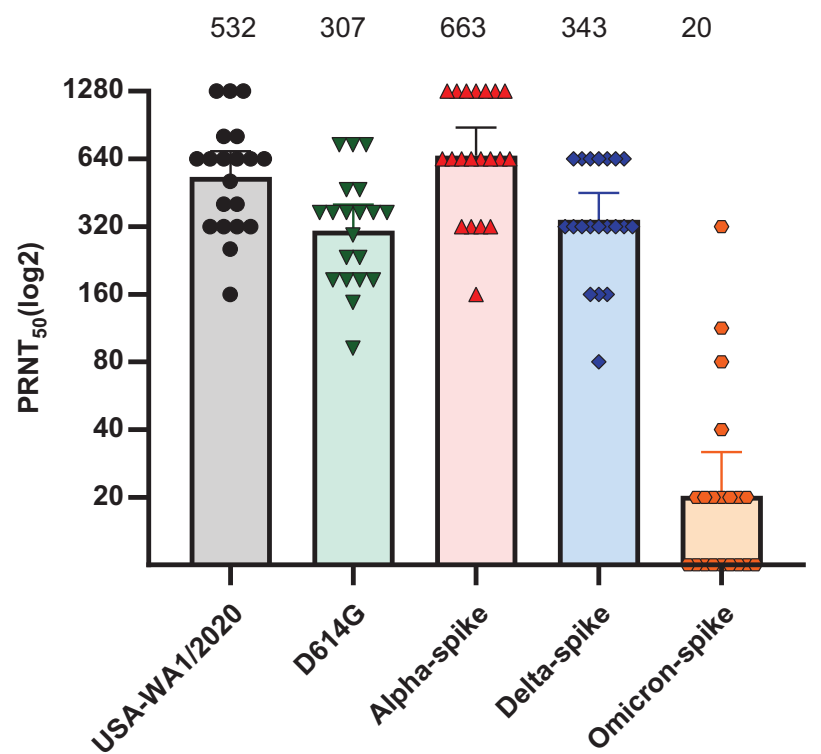

FIGURE 2 | Serum neutralization titers of different lineages of SARS-COV-2.

The D614G mutation or genes encoding S protein from different lineages of SARS-COV-2 were engineered into the USA-WA1/2020 backbone. The $50 \%$ plaque-reduction neutralization testing $\left(\right.$ PRNT $_{50}$ ) for 20 samples obtained from 15 trial participants after administration of the second dose of the BNT162b2 vaccine is shown. Each data point represents the geometric mean PRNT obtained with a serum sample against the indicated virus. The heights of bars and the numbers over the bars indicate geometric mean titers. The error bars indicate $95 \%$ confidence intervals. 
neutralization level has been found to remain robust as long as 4 months after the third dose of the Pfizer vaccine; however, the durability of neutralization beyond 4 months after the third dose remains to be determined [46]. These results support a two-pronged vaccine strategy against Omicron and other newly emerged variants, involving (i) booster vaccination with the currently approved safe vaccines and (ii) modification of the vaccine $\mathrm{S}$ sequences to match those of Omicron and new variants. The mRNA vaccine technology allows for rapid modification of the S sequence. Realworld vaccine effectiveness data and laboratory studies are needed to guide the implementation of this two-pronged vaccine strategy.

Two years have elapsed since the onset of the COVID-19 pandemic, and various SARS-CoV-2 variants, in turns, have dominated viral transmission and surges. The current Omicron surge may not be the last. Although some variants have become able to escape immune protection from vaccination and/or natural infection [43-45,48], compelling evidence demonstrates that vaccination minimizes the risk of severe disease, and lowers the rates of hospitalization and death $[49,50]$. Thus, mass immunization and administration of booster shots with highly effective and safe vaccines, together with mask wearing and social distancing, will continue to be the most effective strategies to finally end the COVID-19 pandemic.

\section{ACKNOWLEDGMENTS}

We thank our colleagues at the University of Texas Medical Branch (UTMB) for helpful discussions. P.-Y.S. was supported by NIH grants HHSN272201600013C, Al134907, Al145617, and UL1TR001439, and awards from the Sealy \& Smith Foundation, the Kleberg Foundation, the John S. Dunn Foundation, the Amon G. Carter Foundation, the Gilson Longenbaugh Foundation, and the Summerfield Robert Foundation.

\section{COMPETING INTERESTS}

P.-Y.S. has filed a patent on the reverse genetic system of SARSCoV-2. The laboratory of P.-Y.S. has received funding support in sponsored research agreements from Pfizer, Gilead, Novartis, GSK, Merck, IGM Biosciences, and Atea Pharmaceuticals. P.-Y.S. is a member of the Pfizer COVID Antiviral Medical Board, a member of the Scientific Advisory Board of Ablmmune, and a founder of FlaviTech.

\section{REFERENCES}

1. WHO Coronavirus (COVID-19) Dashboard. [accessed 2022 Jan 04]. Available from: https://covid19.who.int.

2. Johns Hopkins Coronavirus Resource Center. [accessed 2022 Jan 04]. Available from: https://coronavirus.jhu.edu/.

3. AstraZeneca. The natural evolution of SARS-CoV-2: how science responds to these challenges. [accessed 2022 Jan 04]. Available from: https://www.astrazeneca.com/what-science-can-do/ topics/disease-understanding/the-natural-evolution-of-sarscov-2.html.

4. Korber B, Fischer WM, Gnanakaran S, Yoon H, Theiler J, Abfalterer W, et al. Tracking changes in SARS-CoV-2 spike: evidence that D614G increases infectivity of the COVID-19 virus. Cell. 2020;182(4):812-827.

5. Leung K, Shum MH, Leung GM, Lam TT, Wu JT. Early transmissibility assessment of the N501Y mutant strains of
SARS-CoV-2 in the United Kingdom, October to November 2020. Euro Surveill. 2021;26(1):2002106.

6. Claro IM, da Silva Sales FC, Ramundo MS, Candido DS, Silva CAM, de Jesus JG, et al. Local transmission of SARS-CoV-2 lineage B.1.1.7, Brazil, December 2020. Emerg Infect Dis. 2021;27(3):970-972.

7. Liu Y, Liu J, Plante KS, Plante JA, Xie X, Zhang X, et al. The N501Y spike substitution enhances SARS-CoV-2 infection and transmission. Nature. 2021. Online ahead of print. DOI: https://doi.org/10.1038/s41586-021-04245-0.

8. Kannan SR, Spratt AN, Cohen AR, Naqvi SH, Chand HS, Quinn TP, et al. Evolutionary analysis of the Delta and Delta Plus variants of the SARS-CoV-2 viruses. J Autoimmun. 2021;124:102715.

9. Mlcochova P, Kemp SA, Dhar MS, Papa G, Meng B, Ferreira I, et al. SARS-CoV-2 B.1.617.2 Delta variant replication and immune evasion. Nature. 2021;599(7883):114-119.

10. Liu Y, Liu J, Johnson BA, Xia H, Ku Z, Schindewolf C, et al. Delta spike P681R mutation enhances SARS-CoV-2 fitness over Alpha variant. bioRxiv. 2021.

11. Karim SSA, Karim QA. Omicron SARS-CoV-2 variant: a new chapter in the COVID-19 pandemic. Lancet. 2021;398(10317): 2126-2128.

12. Saxena SK, Kumar S, Ansari S, Paweska JT, Maurya VK, Tripathi AK, et al. Characterization of the novel SARS-CoV-2 Omicron (B.1.1.529) variant of concern and its global perspective. J Med Virol. 2021. Online ahead of print. DOI: https://doi.org/10.1002/ jmv. 27524.

13. Sheward DJ, Kim C, Ehling RA, Pankow A, Dopico XC, Martin $D$, et al. Variable loss of antibody potency against SARS-CoV-2 B.1.1.529 (Omicron). bioRxiv. 2021.

14. Cele S, Jackson L, Khan K, Khoury D, Moyo-Gwete T, Tegally H, et al. SARS-CoV-2 Omicron has extensive but incomplete escape of Pfizer BNT162b2 elicited neutralization and requires ACE2 for infection. medRxiv. 2021.

15. Wilhelm A, Widera M, Grikscheit K, Toptan T, Schenk B, Pallas $C$, et al. Reduced neutralization of SARS-CoV-2 Omicron variant by vaccine sera and monoclonal antibodies. medRxiv. 2021.

16. Cameroni E, Saliba C, Bowen JE, Rosen LE, Culap K, Pinto D, et al. Broadly neutralizing antibodies overcome SARS-CoV-2 Omicron antigenic shift. bioRxiv. 2021.

17. Zou J, Xia H, Xie X, Kurhade C, Machado RRG, Weaver SC, et al. Neutralization against Omicron SARS-CoV-2 from previous non-Omicron infection. bioRxiv 2021. DOI: https://doi.org/10.1101/2021.12.20.473584.

18. Pfizer and BioNTech Provide Update on Omicron Variant. [accessed 2022 Jan 04]. Available from: https://www. pfizer.com/news/press-release/press-release-detail/ pfizer-and-biontech-provide-update-omicron-variant.

19. Walls AC, Park YJ, Tortorici MA, Wall A, McGuire AT, Veesler D. Structure, function, and antigenicity of the SARS-CoV-2 spike glycoprotein. Cell. 2020;181(2):281-292.

20. Bestle D, Heindl MR, Limburg H, Van Lam van T, Pilgram O, Moulton $\mathrm{H}$, et al. TMPRSS2 and furin are both essential for proteolytic activation of SARS-CoV-2 in human airway cells. Life Sci Alliance. 2020;3(9):e202000786.

21. Zhou B, Thao TTN, Hoffmann D, Taddeo A, Ebert N, Labroussaa F, et al. SARS-CoV-2 spike D614G change enhances replication and transmission. Nature. 2021;592(7852):122-127.

22. Plante JA, Liu Y, Liu J, Xia H, Johnson BA, Lokugamage KG, et al. Spike mutation D614G alters SARS-CoV-2 fitness. Nature. 2021;592(7852):116-121.

23. Huang H, Zhu Y, Niu Z, Zhou L, Sun Q. SARS-CoV-2 N501Y variants of concern and their potential transmission by mouse. Cell Death Differ. 2021;28(10):2840-2842.

24. Saito A, Irie T, Suzuki R, Maemura T, Nasser H, Uriu K, et al. Enhanced fusogenicity and pathogenicity of SARS-CoV-2 Delta P681R mutation. Nature. 2021. Online ahead of print. DOI: 10.1038/s41586-021-04266-9. 
25. McCallum M, Bassi J, De Marco A, Chen A, Walls AC, Di lulio J, et al. SARS-CoV-2 immune evasion by the B.1.427/B.1.429 variant of concern. Science. 2021;373(6555):648-654.

26. McCallum M, De Marco A, Lempp FA, Tortorici MA, Pinto D, Walls $A C$, et al. N-terminal domain antigenic mapping reveals a site of vulnerability for SARS-CoV-2. Cell. 2021;184(9): 2332-2347.e2316.

27. McCarthy KR, Rennick LJ, Nambulli S, Robinson-McCarthy LR, Bain WG, Haidar G, et al. Recurrent deletions in the SARSCoV-2 spike glycoprotein drive antibody escape. Science. 2021;371(6534):1139-1142.

28. Ramanathan M, Ferguson ID, Miao W, Khavari PA. SARS-CoV-2 B.1.1.7 and B.1.351 spike variants bind human ACE2 with increased affinity. Lancet Infect Dis. 2021;21(8):1070.

29. Thomson EC, Rosen LE, Shepherd JG, Spreafico R, da Silva Filipe A, Wojcechowskyj JA, et al. Circulating SARS-CoV-2 spike N439K variants maintain fitness while evading antibodymediated immunity. Cell. 2021;184(5):1171-1187.

30. Tandel D, Gupta D, Sah V, Harinivas Harshan K. N440K variant of SARS-COV-2 has higher infectious fitness. bioRxiv. 2021.

31. Starr TN, Greaney AJ, Dingens AS, Bloom JD. Complete map of SARS-CoV-2 RBD mutations that escape the monoclonal antibody LY-CoV555 and its cocktail with LY-CoV016. Cell Rep Med. 2021;2(4):100255

32. Garcia-Beltran WF, Lam EC, St Denis K, Nitido AD, Garcia ZH, Hauser BM, et al. Multiple SARS-CoV-2 variants escape neutralization by vaccine-induced humoral immunity. Cell. 2021;184(9):2523.

33. Zhang W, Davis BD, Chen SS, Sincuir Martinez JM, Plummer JT, Vail E. Emergence of a novel SARS-CoV-2 variant in Southern California. J Am Med Assoc. 2021;325(13):1324-1326.

34. Hoffmann M, Zhang L, Kruger N, Graichen L, Kleine-Weber $\mathrm{H}$, Hofmann-Winkler $\mathrm{H}$, et al. SARS-CoV-2 mutations acquired in mink reduce antibody-mediated neutralization. Cell Rep. 2021;35(3):109017.

35. Gaebler C, Wang Z, Lorenzi JCC, Muecksch F, Finkin S, Tokuyama M, et al. Evolution of antibody immunity to SARSCoV-2. Nature. 2021;591(7851):639-644.

36. Koenig PA, Das H, Liu H, Kummerer BM, Gohr FN, Jenster LM, et al. Structure-guided multivalent nanobodies block SARSCoV-2 infection and suppress mutational escape. Science. 2021;371(6530).

37. Greaney AJ, Starr TN, Gilchuk P, Zost SJ, Binshtein E, Loes AN, et al. Complete mapping of mutations to the SARS-CoV-2 spike receptor-binding domain that escape antibody recognition. Cell Host Microbe. 2021;29(1):44-57.e49.

38. Braun KM, Moreno GK, Halfmann PJ, Hodcroft EB, Baker DA, Boehm EC, et al. Transmission of SARS-CoV-2 in domestic cats imposes a narrow bottleneck. PLoS Pathog. 2021;17:e1009373.

39. GISAID. Tracking of variants. [accessed 2022 Jan 04]. Available from: https://www. gisaid.org/hcov19variants/.

40. Kaur SP, Gupta V. COVID-19 vaccine: a comprehensive status report. Virus Res. 2020;288:198114.

41. Polack FP, Thomas SJ, Kitchin N, Absalon J, Gurtman A, Lockhart S, et al. Safety and efficacy of the BNT162b2 mRNA COVID-19 vaccine. N Engl J Med. 2020;383(27):2603-2615.

42. Liu Y, Liu J, Xia H, Zhang X, Fontes-Garfias CR, Swanson KA, et al. Neutralizing activity of BNT162b2-elicited serum. N Engl J Med. 2021;384(15):1466-1468.

43. Xie X, Muruato A, Lokugamage KG, Narayanan $K$, Zhang $X$, Zou J, et al. An infectious CDNA clone of SARS-CoV-2. Cell Host Microbe. 2020;27(5):841-848.e843.

44. Zou J, Xie X, Fontes-Garfias CR, Swanson KA, Kanevsky I, Tompkins K, et al. The effect of SARS-CoV-2 D614G mutation on BNT162b2 vaccine-elicited neutralization. NPJ Vaccines. 2021;6(1):44.

45. Liu J, Liu Y, Xia H, Zou J, Weaver SC, Swanson KA, et al. BNT162b2-elicited neutralization of B.1.617 and other SARSCoV-2 variants. Nature. 2021;596(7871):273-275.

46. Xia H, Zou J, Kurhade C, Cai H, Yang Q, Cutler M, et al. Neutralization of Omicron SARS-CoV-2 by 2 or 3 doses of BNT162b2 vaccine. bioRxiv. 2022

47. Falsey AR, Frenck RW Jr, Walsh EE, Kitchin N, Absalon J, Gurtman A, et al. SARS-CoV-2 neutralization with BNT162b2 vaccine dose 3. N Engl J Med. 2021;385(17):1627-1629.

48. Xie X, Liu Y, Liu J, Zhang X, Zou J, Fontes-Garfias CR, et al. Neutralization of SARS-CoV-2 spike 69/70 deletion, E484K and N501Y variants by BNT162b2 vaccine-elicited sera. Nat Med. 2021;27(4):620-621.

49. Self WH, Tenforde MW, Rhoads JP, Gaglani M, Ginde AA, Douin DJ, et al. Comparative effectiveness of Moderna, Pfizer-BioNTech, and Janssen (Johnson \& Johnson) vaccines in preventing COVID-19 hospitalizations among adults without immunocompromising conditions - United States, March-August 2021. MMWR Morb Mortal Wkly Rep. 2021;70(38):1337-1343

50. Cohn BA, Cirillo PM, Murphy CC, Krigbaum NY, Wallace AW. SARS-COV-2 vaccine protection and deaths among US veterans during 2021. Science. 2021;eabm0620. 